\title{
MAXIMIZING DIVIDENDS WITHOUT BANKRUPTCY
}

BY

\author{
Hans U. Gerber, Elias S.W. Shiu and Nathaniel Smith
}

\begin{abstract}
Consider the classical compound Poisson model of risk theory, in which dividends are paid to the shareholders according to a barrier strategy. Let $b^{*}$ be the level of the barrier that maximizes the expectation of the discounted dividends until ruin. This paper is inspired by Dickson and Waters (2004). They point out that the shareholders should be liable to cover the deficit at ruin. Thus, they consider $b^{o}$, the level of the barrier that maximizes the expectation of the difference between the discounted dividends until ruin and the discounted deficit at ruin. In this paper, $b^{*}$ and $b^{o}$ are compared, when the claim amount distribution is exponential or a combination of exponentials.
\end{abstract}

\section{KEYWORDS}

Dividends, barrier strategies, Dickson and Waters modification, mixtures and combinations of exponential claim amount distributions.

\section{INTRODUCTION}

In classical risk theory, the probability of ruin and related quantities are calculated. In this model, due to the loading contained in the premiums, the surplus drifts to infinity in the long run (at least in the typical case where ruin does not occur). De Finetti (1957) remarked that this is not realistic. He considered a stock company. Then, according to De Finetti, the goal of the company is to maximize the expectation of the discounted dividends to the shareholders until possible ruin. In mathematical terminology, this leads to a problem of optimal control. But the economic aspect of this model is of equal importance. Borch $(1974,1990)$ was an active participant of the early ASTIN Colloquia; he contributed to the dissemination and development of De Finetti's idea.

The problem of maximizing the expectation of the discounted dividends is covered in the monographs of Bühlmann (1970), Gerber (1979) and Dickson (2005). All three consider the problem in the classical compound Poisson model of risk theory. Barrier strategies play an important role. If a barrier strategy with parameter $b$ is applied, no dividends are paid while the surplus is below $b$, and the overflow with respect to the level $b$ is paid as dividends. Let $b^{*}$ denote 
the optimal value of $b$. If the initial surplus is less than $b^{*}$, the barrier strategy with parameter $b^{*}$ is the best strategy among all strategies ${ }^{1}$.

If a barrier strategy is applied, ruin will take place with certainty. At the time of ruin, there is a deficit, and part of the last claim is not covered. Hence ruin implies bankruptcy. This is perfectly in line with economic theory. However, in their paper rich of ideas, Dickson and Waters (2004) argue that an insurance company should behave in a more responsible way. They postulate that the shareholders should be liable to cover the deficit at the time of ruin, thereby avoiding outright bankruptcy. This leads to a new mathematical problem, the maximization of the expectation of the difference between discounted dividends until ruin and the deficit at ruin. As a consequence, there is a modified optimal barrier, which is denoted by $b^{o}$. Dickson and Waters (2004) calculate $b^{o}$ in certain cases. The goal of this paper is to analyze in more detail the difference between $b^{*}$ and $b^{o}$. An effort has been made to present a self-contained methodology.

\section{THE CLASSICAL PROBLEM}

In the absence of dividends, the surplus of an insurance company at time $t$ is

$$
U(t)=U(0)+c t-S(t), \quad t \geq 0 .
$$

The premiums are received continuously at a constant rate $c$, and the aggregate claims process $\{S(t)\}$ is a compound Poisson process, specified by the claim frequency $\lambda$ and the probability density function $p(y)$ of the individual claim amounts. The relative security loading $\theta$ is defined by the equation

$$
c=(1+\theta) \lambda p_{1},
$$

where

$$
p_{1}=\int_{0}^{\infty} y p(y) d y
$$

is the expected claim size. We suppose that $\theta$ is positive.

Dividends are paid according to some dividend strategy. Let $D(t)$ denote the aggregate dividends by time $t$. Then

$$
X(t)=U(t)-D(t)
$$

is the surplus of the company at time $t$, and

$$
T=\inf \{t: X(t)<0\}
$$

is the time of ruin.

1 If the initial surplus is greater than $b^{*}$, the optimal strategy may be much more complicated; the reader may want to consult the book by Schmidli (2007) and also Azcue and Muler (2005). 
The classical problem is to maximize the expectation of $\int_{0}^{T} e^{-\delta t} d D(t)$, the discounted dividends until ruin, where $\delta>0$ is a force of interest. In this paper, we only consider barrier strategies for dividend payments. If dividends are paid according to a barrier strategy with parameter $b>0$, no dividends are paid whenever $X(t)<b$, and dividends at the rate $c$ are paid whenever $X(t)=b$. We assume $X(0)=x \leq b$; then $X(t)$ will never exceed $b$. Let $V(x ; b), 0 \leq x \leq b$, denote the expectation of the discounted dividends, if the barrier strategy with parameter $b$ is applied. As a function of $x, V(x ; b)$ satisfies the integro-differential equation

$$
c V^{\prime}(x ; b)-(\lambda+\delta) V(x ; b)+\lambda \int_{0}^{x} V(y ; b) p(x-y) d y=0, \quad 0<x<b .
$$

Moreover, $V(x ; b)$ satisfies the boundary condition

$$
V^{\prime}(b ; b)=1 .
$$

It follows that

$$
V(x ; b)=\frac{h(x)}{h^{\prime}(b)}, \quad 0 \leq x \leq b,
$$

where $h(x)$ is a positive solution of the equation

$$
c h^{\prime}(x)-(\lambda+\delta) h(x)+\lambda \int_{0}^{x} h(y) p(x-y) d y=0, x>0 .
$$

Let $b^{*}$ denote the optimal value of $b$, that is, the value that maximizes $V(x ; b)$ for a given $x, x<b$. From (2.8) we see that $b^{*}$ is the value of $b$ which minimizes $h^{\prime}(b)$. We assume that $b^{*}>0$. It follows that

$$
h^{\prime \prime}\left(b^{*}\right)=0
$$

and that

$$
V^{\prime \prime}\left(b^{*} ; b^{*}\right)=0
$$

If $X(0)=x \leq b^{*}$, the barrier strategy with parameter $b^{*}$ is optimal among all dividend strategies.

Equations (2.6) and (2.9) can be written in an alternative form, if we substitute for $c$ according to (2.2) and divide the resulting equation by $\lambda$. For example, (2.6) becomes the equation

$$
(1+\theta) p_{1} V^{\prime}(x ; b)-(1+\alpha) V(x ; b)+\int_{0}^{x} V(y ; b) p(x-y) d y=0, \quad 0<x<b,
$$


with

$$
\alpha=\frac{\delta}{\lambda}
$$

This shows that for a given claim amount distribution, the two relevant parameters are $\theta$ and $\alpha$.

\section{THE DEFICIT AT RUIN}

The deficit at ruin is $|X(T)|$. Let $R(x ; b)$ denote the expectation of the discounted deficit at ruin, if the barrier strategy with parameter $b$ is applied. As a function of $X(0)=x, R(x ; b)$ satisfies the integro-differential equation

$$
\begin{aligned}
& c R^{\prime}(x ; b)-(\lambda+\delta) R(x ; b) \\
& +\lambda \int_{0}^{x} R(y ; b) p(x-y) d y \\
& \quad+\lambda \int_{x}^{\infty}[1-P(y)] d y=0, \quad 0<x<b,
\end{aligned}
$$

together with the boundary condition

$$
R^{\prime}(b ; b)=0
$$

We note that (3.1) and (3.2) are special cases of (2.6) and (2.8) of Lin, Willmot and Drekic (2003). To keep this paper self-contained, we provide a proof of (3.1) and (3.2). Suppose $x<b$ and $h<\frac{b-x}{c}$. By conditioning on the time and the amount of the first claim in the interval $(0, h)$ we see that

$$
\begin{aligned}
R(x ; b) & =e^{-(\lambda+\delta) h} R(x+c h ; b) \\
& +\lambda \int_{0}^{h} e^{-(\lambda+\delta) t} \int_{0}^{x+c t} R(x+c t-y ; b) p(y) d y d t \\
& +\lambda \int_{0}^{h} e^{-(\lambda+\delta) t} \int_{x+c t}^{\infty}(y-x-c t) p(y) d y d t .
\end{aligned}
$$

Now we differentiate this equation with respect to $h$ and set $h=0$ in the resulting equation. This yields

$$
\begin{aligned}
0 & =c R^{\prime}(x ; b)-(\lambda+\delta) R(x ; b) \\
& +\lambda \int_{0}^{x} R(x-y ; b) p(y) d y \\
& +\lambda \int_{x}^{\infty}(y-x) p(y) d y .
\end{aligned}
$$


The first integral is a convolution integral. If we change the variable in the convolution integral and integrate the second integral by parts, we obtain (3.1). Now suppose $X(0)=b$, and let $h>0$. Then

$$
\begin{aligned}
R(b ; b) & =e^{-(\lambda+\delta) h} R(b ; b) \\
& +\lambda \int_{0}^{h} e^{-(\lambda+\delta) t} \int_{0}^{b} R(b-y ; b) p(y) d y d t \\
& +\lambda \int_{0}^{h} e^{-(\lambda+\delta) t} \int_{b}^{\infty}(y-b) p(y) d y d t .
\end{aligned}
$$

Differentiating this equation with respect to $h$ and setting $h=0$ yields

$$
0=-(\lambda+\delta) R(b ; b)+\lambda \int_{0}^{b} R(b-y ; b) p(y) d y+\lambda \int_{b}^{\infty}(y-b) p(y) d y .
$$

A comparison of (3.4) with $x=b$ and (3.6) leads to (3.2).

We remark that equation (3.1) can be written alternatively as

$$
\begin{aligned}
& (1+\theta) p_{1} R^{\prime}(x ; b)-(1+\alpha) R(x ; b) \\
& +\int_{0}^{x} R(y ; b) p(x-y) d y \\
& \quad+\int_{x}^{\infty}[1-P(y)] d y=0,0<x<b .
\end{aligned}
$$

\section{The Dickson-Waters MOdification}

Dickson and Waters (2004) brought up the idea that the shareholders should be held responsible to cover the deficit at ruin. If this is the case, the shareholders will want to maximize the expectation of the difference of the discounted dividends until ruin and the discounted deficit at ruin.

Again we consider a barrier strategy with parameter $b$. Then $b$ is chosen to maximize

$$
W(x ; b)=V(x ; b)-R(x ; b)
$$

for given $x<b$. We denote the optimal value of $b$ by $b^{o}$ and assume $b^{o}>0$. It follows that

$$
\left.\frac{\partial}{\partial b} W(x ; b)\right|_{b=b^{\circ}}=0
$$

Note that $b^{o}$ does not depend on $x$. 
From (2.7) and (3.2) we gather that

$$
W^{\prime}(b ; b)=1 .
$$

Now we differentiate this equation with respect to $b$ to see that

$$
W^{\prime \prime}(b ; b)+\left.\frac{\partial}{\partial b} W^{\prime}(x ; b)\right|_{x=b}=0
$$

From this and (4.2) it follows that

$$
W^{\prime \prime}\left(b^{o} ; b^{o}\right)=0 .
$$

The optimality conditions (4.5) and (2.11) are known as high contact conditions in finance literature.

\section{EXPONENTIAL CLAIM AMOUNT DISTRIBUTION}

We discuss the special case where

$$
p(y)=\beta e^{-\beta y}, y>0,
$$

where $p_{1}=1 / \beta$.

It has the merit that many calculations can be performed explicitly. We note that $p^{\prime}(y)=-\beta p(y)$, from which it follows that

$$
\left(\frac{d}{d x}+\beta\right) \int_{0}^{x} V(y ; b) p(x-y) d y=\beta V(x ; b) .
$$

Thus, if we apply the operator $\left(\frac{d}{d x}+\beta\right)$ to the integro-differential equation (2.12), we obtain a differential equation for the function $V(x ; b)$ :

$$
(1+\theta) p_{1} V^{\prime \prime}(x ; b)+(\theta-\alpha) V^{\prime}(x ; b)-\alpha \beta V(x ; b)=0 .
$$

Similarly, if we apply the operator $\left(\frac{d}{d x}+\beta\right)$ to (3.7), we obtain the same differential equation for the function $R(x ; b)$. We conclude that

$$
\begin{aligned}
& V(x ; b)=C_{0} e^{r x}+C_{1} e^{s x}, 0 \leq x \leq b, \\
& R(x ; b)=D_{0} e^{r x}+D_{1} e^{s x}, 0 \leq x \leq b,
\end{aligned}
$$

where $r>0$ and $s<0$ are the solutions of the characteristic equation

$$
(1+\theta) p_{1} \xi^{2}+(\theta-\alpha) \xi-\alpha \beta=0,
$$


and the coefficients $C_{k}=C_{k}(b), D_{k}=D_{k}(b), k=0,1$, have yet to be determined.

Formula (2.7) leads to the condition

$$
C_{0} r e^{r b}+C_{1} s e^{s b}=1 .
$$

Substituting (5.4) in (2.12) we get a second condition:

$$
\frac{C_{0}}{\beta+r}+\frac{C_{1}}{\beta+s}=0 \text {. }
$$

From (5.7) and (5.8) we find that

$$
\begin{aligned}
& C_{0}=\frac{\beta+r}{v(b),} \\
& C_{1}=-\frac{\beta+s}{v(b)},
\end{aligned}
$$

with

$$
v(b)=r(\beta+r) e^{r b}-s(\beta+s) e^{s b} .
$$

The condition $v^{\prime}\left(b^{*}\right)=0$ yields

$$
b^{*}=\frac{1}{r-s} \ln \frac{s^{2}(\beta+s)}{r^{2}(\beta+r)} .
$$

We note that condition (2.11) leads to the same expression for $b^{*}$.

Formula (3.2) implies that

$$
D_{0} r e^{r b}+D_{1} s e^{s b}=0 .
$$

Substituting (5.5) in (3.7), we obtain the second condition to determine $D_{0}$ and $D_{1}$ :

$$
\frac{\beta^{2}}{\beta+r} D_{0}+\frac{\beta^{2}}{\beta+s} D_{1}=1 \text {. }
$$

Thus we find that

$$
\begin{gathered}
D_{0}=-s e^{s b} \frac{(\beta+r)(\beta+s)}{\beta^{2} v(b)}, \\
D_{1}=r e^{r b} \frac{(\beta+r)(\beta+s)}{\beta^{2} v(b)},
\end{gathered}
$$

with $v(b)$ given by (5.11). 
For the Dickson-Waters modification, the function to be maximized is

$$
W(x ; b)=\left(C_{0}(b)-D_{0}(b)\right) e^{r x}+\left(C_{1}(b)-D_{1}(b)\right) e^{s x} .
$$

Then the condition for $b^{o}$ is that

$$
\frac{\partial W}{\partial b}=\left(C_{0}^{\prime}(b)-D_{0}^{\prime}(b)\right) e^{r x}+\left(C_{1}^{\prime}(b)-D_{1}^{\prime}(b)\right) e^{s x}=0 .
$$

Thus $b^{o}$ satisfies the conditions

$$
\begin{aligned}
& C_{0}^{\prime}(b)-D_{0}^{\prime}(b)=0, \\
& C_{1}^{\prime}(b)-D_{1}^{\prime}(b)=0 .
\end{aligned}
$$

These two conditions are actually equivalent. From (5.8) and (5.14) it follows that

$$
\frac{\beta^{2}}{\beta+r}\left(C_{0}-D_{0}\right)+\frac{\beta^{2}}{\beta+s}\left(C_{1}-D_{1}\right)=-1 \text {. }
$$

Hence

$$
\frac{1}{\beta+r}\left(C_{0}^{\prime}-D_{0}^{\prime}\right)+\frac{1}{\beta+s}\left(C_{1}^{\prime}-D_{1}^{\prime}\right)=0 .
$$

This shows that (5.19) and (5.20) are indeed equivalent. There is another way to obtain $b^{o}$. From (4.5) it follows that $b^{o}$ satisfies the equation

$$
r^{2}\left(C_{0}\left(b^{o}\right)-D_{0}\left(b^{o}\right)\right) e^{r b^{o}}+s^{2}\left(C_{1}\left(b^{o}\right)-D_{1}\left(b^{o}\right)\right) e^{s b^{o}}=0 .
$$

The three equations (5.19), (5.20) and (5.23) are implicit equations for $b^{o}$; they must be solved numerically.

Remark: If we set $x=b=b^{*}$ in the differential equation (5.3), we find from (2.7) and (2.11) that

$$
V\left(b^{*} ; b^{*}\right)=\frac{\theta}{\alpha \beta}-\frac{1}{\beta} .
$$

Now $W(x ; b)$ satisfies the same differential equation as $V(x ; b)$. Hence it follows from (4.3) and (4.5) that

$$
W\left(b^{o} ; b^{o}\right)=\frac{\theta}{\alpha \beta}-\frac{1}{\beta} .
$$




\section{A FIRST COMPARISON OF $b^{*}$ AND $b^{o}$}

One might wonder, how the Dickson-Waters modification affects the optimal value of the dividend barrier. To find a first answer, we assume an exponential claim amount probability density as in the preceding section. It is also judicious to scale the monetary unit, so that $\beta=1$ in the new monetary unit.

Equation (5.6) simplifies to

$$
(1+\theta) \xi^{2}+(\theta-\alpha) \xi-\alpha=0 .
$$

There remains two relevant parameters in this model, $\theta$ and $\alpha$. Thus $r$ and $s, C_{0}$, $C_{1}, D_{0}, D_{1}$ and last but not least, $b^{*}$ and $b^{o}$ depend only on these two parameters.

For $b^{*}$ there is the explicit expression (5.12). In contrast, $b^{o}$ is obtained by an algorithm, for example by the following iteration. From (5.23) it follows that $b^{o}$ is the solution of the equation

$$
b=\frac{1}{r-s} \ln \left(\frac{s^{2}\left(D_{1}(b)-C_{1}(b)\right)}{r^{2}\left(C_{0}(b)-D_{0}(b)\right)}\right) .
$$

We denote the expression on the right hand side of (6.2) by $g(b)$. Hence, we calculate $b_{1}, b_{2}, b_{3}, \ldots$ recursively by the formula

$$
b_{k+1}=g\left(b_{k}\right) \text {. }
$$

We observe that the $b_{k}$ 's converge rapidly to $b^{o}$. There is an explanation for the rapid convergence: from (5.19) and (5.20) it follows that $g^{\prime}\left(b^{o}\right)=0$, which implies at least quadratic convergence, see for example theorem 2.9 in Burden et al. (1981).

Table 1 exhibits the values of $b^{*}$ and Table 2 shows the corresponding values of $b^{o}$. We observe that $b^{o}>b^{*}$ consistently. There is a theoretical justification for this: Suppose that $b^{*}>0$; we show that $b^{o}>b^{*}$.

Under the assumption $b^{*}>0$, equation (2.10) has the unique solution (5.12). It follows that $h^{\prime \prime}(b)<0$ for $0<b<b^{*}$ and hence

$$
\frac{\partial}{\partial b} V(x ; b)>0, x<b<b^{*} .
$$

Now consider

$$
L(x ; b)=\mathbf{E}\left[e^{-\delta T} \mid X(0)=x\right],
$$

the expected present value of a payment of one at the time of ruin. This function has been examined by Paulsen and Gjessing (1997), Lin, Willmot and Drekic (2003) and Gerber and Shiu (2006). It is clear that

$$
\frac{\partial}{\partial b} L(x ; b)<0 \text {. }
$$




\begin{tabular}{rrrrrrrrr|}
\hline$\underline{\theta}$ & $\mathbf{0 0 0 1}$ & $\mathbf{. 0 0 1}$ & .01 & $\mathbf{. 0 2 5}$ & .05 & .10 & .20 \\
\hline 10 & 96.57 & 42.91 & 6.99 & 1.86 & 0.00 & 0.00 & 0.00 \\
.20 & 68.29 & 39.39 & 11.89 & 4.92 & 1.74 & 0.00 & 0.00 \\
.30 & 55.42 & 34.87 & 13.82 & 6.93 & 3.20 & .78 & 0.00 \\
.40 & 48.05 & 31.59 & 14.47 & 8.16 & 4.31 & 1.55 & 0.00 \\
.50 & 43.26 & 29.22 & 14.60 & 8.90 & 5.14 & 2.21 & .24 \\
.60 & 39.89 & 27.46 & 14.53 & 9.35 & 5.74 & 2.77 & .63 \\
.70 & 37.39 & 26.09 & 14.39 & 9.62 & 6.20 & 3.23 & .98 \\
.80 & 35.46 & 25.01 & 14.22 & 9.79 & 6.54 & 3.62 & 1.29 \\
.90 & 33.92 & 24.13 & 14.05 & 9.90 & 6.80 & 3.94 & 1.58 \\
1.00 & 32.67 & 23.41 & 13.89 & 9.96 & 7.00 & 4.21 & 1.83 \\
1.10 & 31.64 & 22.80 & 13.74 & 10.01 & 7.17 & 4.45 & 2.06 \\
1.20 & 30.77 & 22.28 & 13.61 & 10.03 & 7.30 & 4.65 & 2.27 \\
1.30 & 30.02 & 21.84 & 13.48 & 10.04 & 7.40 & 4.82 & 2.46 \\
1.40 & 29.38 & 21.45 & 13.37 & 10.05 & 7.49 & 4.97 & 2.63 \\
1.50 & 28.82 & 21.12 & 13.27 & 10.05 & 7.57 & 5.10 & 2.79 \\
1.60 & 28.33 & 20.82 & 13.18 & 10.05 & 7.63 & 5.22 & 2.93 \\
1.70 & 27.89 & 20.55 & 13.10 & 10.05 & 7.69 & 5.32 & 3.06 \\
1.80 & 27.50 & 20.32 & 13.03 & 10.04 & 7.74 & 5.42 & 3.18 \\
1.90 & 27.16 & 20.11 & 12.96 & 10.04 & 7.78 & 5.50 & 3.29 \\
2.00 & 26.84 & 19.92 & 12.90 & 10.03 & 7.82 & 5.58 & 3.39 \\
& & & & & & & \\
\hline
\end{tabular}

TABLE 1: $b^{*}$ if $p(x)=e^{-x}, x>0$.

\begin{tabular}{|c|c|c|c|c|c|c|c|}
\hline$\underline{\theta}$ & .0001 & .001 & .01 & .025 & $\begin{array}{c}\alpha \\
.05\end{array}$ & .10 & .20 \\
\hline .10 & 96.58 & 43.05 & 7.68 & 2.72 & .88 & 0.00 & 0.00 \\
\hline .20 & 68.30 & 39.43 & 12.24 & 5.53 & 2.52 & .78 & 0.00 \\
\hline .30 & 55.42 & 34.88 & 14.01 & 7.34 & 3.80 & 1.55 & .24 \\
\hline .40 & 48.05 & 31.60 & 14.58 & 8.43 & 4.77 & 2.22 & .63 \\
\hline .50 & 43.26 & 29.23 & 14.67 & 9.09 & 5.49 & 2.77 & .98 \\
\hline .60 & 39.89 & 27.46 & 14.58 & 9.49 & 6.02 & 3.24 & 1.29 \\
\hline .70 & 37.39 & 26.09 & 14.43 & 9.73 & 6.42 & 3.63 & 1.58 \\
\hline .80 & 35.46 & 25.01 & 14.25 & 9.88 & 6.72 & 3.96 & 1.84 \\
\hline .90 & 33.92 & 24.13 & 14.08 & 9.97 & 6.95 & 4.24 & 2.07 \\
\hline 1.00 & 32.67 & 23.41 & 13.91 & 10.03 & 7.13 & 4.47 & 2.28 \\
\hline 1.10 & 31.64 & 22.80 & 13.76 & 10.06 & 7.28 & 4.67 & 2.47 \\
\hline 1.20 & 30.77 & 22.28 & 13.62 & 10.07 & 7.39 & 4.85 & 2.64 \\
\hline 1.30 & 30.02 & 21.84 & 13.50 & 10.08 & 7.49 & 5.00 & 2.80 \\
\hline 1.40 & 29.38 & 21.45 & 13.39 & 10.08 & 7.57 & 5.13 & 2.94 \\
\hline 1.50 & 28.82 & 21.12 & 13.29 & 10.08 & 7.64 & 5.25 & 3.07 \\
\hline 1.60 & 28.33 & 20.82 & 13.20 & 10.08 & 7.69 & 5.35 & 3.19 \\
\hline 1.70 & 27.89 & 20.56 & 13.11 & 10.07 & 7.74 & 5.45 & 3.30 \\
\hline 1.80 & 27.50 & 20.32 & 13.04 & 10.07 & 7.79 & 5.53 & 3.40 \\
\hline 1.90 & 27.16 & 20.11 & 12.97 & 10.06 & 7.83 & 5.61 & 3.50 \\
\hline 2.00 & 26.84 & 19.92 & 12.91 & 10.05 & 7.86 & 5.67 & 3.59 \\
\hline
\end{tabular}

TABLE 2: $b^{o}$ if $p(x)=e^{-x}, x>0$. 
In the special case of an exponential claim amount distribution (with $\beta=1$ ) it follows from its memoryless property that

$$
R(x ; b)=L(x ; b) .
$$

Then

$$
\frac{\partial}{\partial b} W(x ; b)=\frac{\partial}{\partial b} V(x ; b)-\frac{\partial}{\partial b} L(x ; b) \geq-\frac{\partial}{\partial b} L(x ; b)>0, x<b \leq b^{*},
$$

from which it follows that $b^{o}>b^{*}$.

Remark 1: Suppose that both $b^{*}$ and $b^{o}$ are positive. From (5.24) and (5.25) it follows that

$$
V\left(b^{*} ; b^{*}\right)=W\left(b^{o} ; b^{o}\right)
$$

which explains again why $b^{o}>b^{*}$.

Remark 2: From formula (1.16) in chapter 10 of Gerber (1979) it follows that $b^{*}$ is positive, if and only if

$$
\alpha<\sqrt{1+\theta}-1
$$

An independent proof is as follows. By using the fact that both $r$ and $s$ are solutions of the quadratic equation (6.1), we obtain an alternative expression for $b^{*}$,

$$
b^{*}=\frac{1}{r-s} \ln \left(\frac{A-B S}{A-B r}\right)
$$

with

$$
\begin{gathered}
A=\alpha(\alpha+1), \\
B=\theta+1-(\alpha+1)^{2} .
\end{gathered}
$$

Note that the expression on the right hand side of (6.11) is positive if and only if $B$ is positive, which is equivalent to condition (6.10).

\section{COMBINATIONS OF EXPONENTIAL DISTRIBUTIONS}

We replace (5.1) by the more general assumption that

$$
p(y)=\sum_{i=1}^{n} A_{i} \beta_{i} e^{-\beta_{i} y}, y>0,
$$


with $0<\beta_{1}<\beta_{2}<\ldots<\beta_{n}$ and $A_{1}+A_{2}+\ldots+A_{n}=1$. We apply the operator

$$
\prod_{i=1}^{n}\left(\frac{d}{d x}+\beta_{i}\right)
$$

to the integro-differential equations (2.6) and (3.1) and use (5.2). We see that $V(x ; b)$ and $R(x ; b)$ satisfy the same homogeneous linear differential equation with constant coefficients of order $n+1$. Thus the functions $V(x ; b)$ and $R(x ; b)$ must be of the same form,

$$
V(x ; b)=\sum_{k=0}^{n} C_{k} e^{\rho_{k} x}, 0 \leq x \leq b,
$$

and

$$
R(x ; b)=\sum_{k=0}^{n} D_{k} e^{\rho_{k} x}, 0 \leq x \leq b .
$$

Substitution of (7.1) and (7.3) in (2.12) yields the condition

$$
\begin{aligned}
(1+\theta) & p_{1} \sum_{k=0}^{n} C_{k} \rho_{k} e^{\rho_{k} x}-(1+\alpha) \sum_{k=0}^{n} C_{k} e^{\rho_{k} x} \\
+ & \sum_{i=1}^{n} \sum_{k=0}^{n} A_{i} C_{k} \frac{\beta_{i}}{\beta_{i}+\rho_{k}}\left(e^{\rho_{k} x}-e^{-\beta_{i} x}\right)=0 .
\end{aligned}
$$

Comparing the coefficients of $e^{\rho_{k} x}$ we obtain the condition

$$
(1+\theta) p_{1} \rho_{k}-(1+\alpha)+\sum_{i=1}^{n} A_{i} \frac{\beta_{i}}{\beta_{i}+\rho_{k}}=0 .
$$

Thus $\rho_{0}, \rho_{1}, \ldots, \rho_{n}$ are the solutions of the equation

$$
(1+\theta) p_{1} \xi-(1+\alpha)+\sum_{i=1}^{n} A_{i} \frac{\beta_{i}}{\beta_{i}+\xi}=0 .
$$

Now we compare the coefficients of $e^{-\beta_{i} x}$ to see that

$$
\sum_{k=0}^{n} C_{k} \frac{1}{\beta_{i}+\rho_{k}}=0, \quad \mathrm{i}=1, \ldots, n .
$$

Furthermore, from (2.7) it follows that

$$
\sum_{k=0}^{n} C_{k} \rho_{k} e^{\rho_{k} b}=1
$$


(7.8) and (7.9) constitute a system of $n+1$ linear equations for $C_{0}, \ldots, C_{n}$.

Substitution of (7.1) and (7.4) in (3.7) yields the condition that

$$
\begin{gathered}
(1+\theta) p_{1} \sum_{k=0}^{n} D_{k} \rho_{k} e^{\rho_{k} x}-(1+\alpha) \sum_{k=0}^{n} D_{k} e^{\rho_{k} x} \\
+\sum_{i=1}^{n} \sum_{k=0}^{n} A_{i} D_{k} \frac{\beta_{i}}{\beta_{i}+\rho_{k}}\left(e^{\rho_{k} x}-e^{-\beta_{i} x}\right) \\
+\sum_{i=1}^{n} \frac{A_{i}}{\beta_{i}} e^{-\beta_{i} x}=0 .
\end{gathered}
$$

Comparing the coefficients of $e^{-\beta_{i} x}$, we see that

$$
\sum_{k=0}^{n} D_{k} \frac{1}{\beta_{i}+\rho_{k}}=\frac{1}{\beta_{i}^{2}}, \quad i=1, \ldots, n .
$$

Furthermore, it follows from (3.2) that

$$
\sum_{k=0}^{n} D_{k} \rho_{k} e^{\rho_{k} b}=0 .
$$

(7.11) and (7.12) constitute a system of $n+1$ linear equations for $D_{0}, \ldots, D_{n}$. It is remarkable that the systems of linear equations for the $C_{k}$ 's and the $D_{k}$ 's have the same coefficient matrix.

In writing (7.3) and (7.4) we exclude tacitly the unlikely situation where some of the solutions of (7.7) coincide. For $n=2$, the three solutions of the equation

$$
A_{1} \frac{\beta_{1}}{\beta_{1}+\xi}+A_{2} \frac{\beta_{2}}{\beta_{2}+\xi}=1+\alpha-(1+\theta) p_{1} \xi
$$

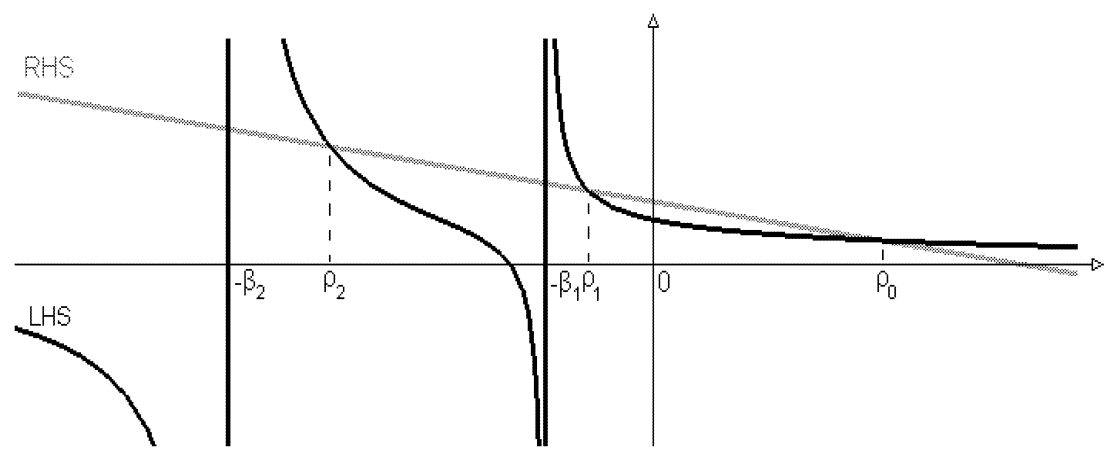

Figure 1: The solutions of (7.13) if $A_{1}>0, A_{2}>0$. 


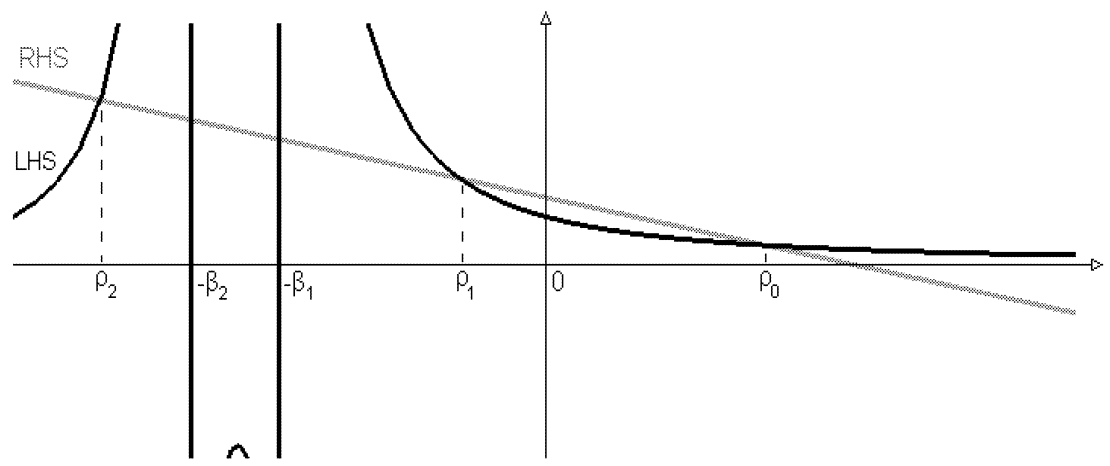

FIgURE 2: The solutions of (7.13) if $A_{1}>0, A_{2}<0$.

are always distinct. In fact,

$$
\rho_{0}>0>\rho_{1}>-\beta_{1}>\rho_{2}>-\beta_{2}
$$

if $A_{1}>0$ and $A_{2}>0$, and

$$
\rho_{0}>0>\rho_{1}>-\beta_{1}>-\beta_{2}>\rho_{2}
$$

if $A_{1}>0$ and $A_{2}<0$. These inequalities are readily confirmed by the graphs in Figure 1 and Figure 2.

\section{FURTHER COMPARISON OF $b^{*}$ AND $b^{o}$}

In Section 5, we calculated $b^{*}$ and $b^{o}$ for the case where $p(x)=e^{-x}, x>0$. We note that this distribution has mean 1 , variance 1 , and a skewness (the ratio of the third central moment to the third power of the standard deviation) of 2.

We shall consider two examples, each of which is a combination of two exponential distributions and has mean 1 .

Example 1: We consider the following mixture of exponential probability densities

$$
p(x)=\frac{1}{3}\left(\frac{1}{2} e^{-\frac{x}{2}}\right)+\frac{2}{3}\left(2 e^{-2 x}\right), x>0 .
$$

Its variance is 2 , and its skewness is 3.36 .

Example 2: We consider

$$
p(x)=2\left(\frac{3}{2} e^{-\frac{3 x}{2}}\right)-1\left(3 e^{-3 x}\right), x>0,
$$



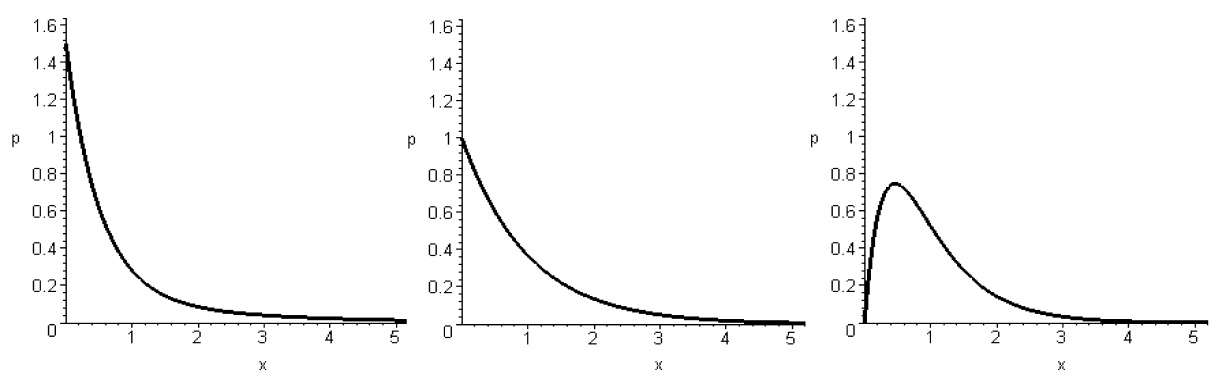

FIGURE 3: The shape of $p(x)$.

which is the convolution of the exponential probability densities of parameters $\frac{3}{2}$ and 3 , respectively. Its variance is $\frac{5}{9}$, and its skewness is 1.61 .

Based on the values of the variance and the skewness, the distribution of Example 1 appears to be more dangerous than the distribution of Example 2, and the exponential distribution is in between. This is illustrated by Figure 3, where the three probability density functions are displayed in decreasing order of "danger".

We calculated the optimal barriers $b^{*}$ and $b^{o}$ with the method explained in Section 7. The results are shown in Tables 3-6. They lead us to the following observations.

a) For the three cases considered, the optimal barriers $b^{*}$ and $b^{\circ}$ are decreasing functions of $\alpha$, but they are not monotone functions of $\theta$.

b) For the three cases, $b^{*}$ and $b^{o}$, as functions of $\alpha$ and $\theta$, follow the same pattern. The difference between $b^{*}$ and $b^{o}$ is small. Note that $b^{*}>b^{o}$ in the upper right hand corner of Tables 3 and 4 .

c) In Tables 5 and $6, b^{*}$ and $b^{\circ}$, as functions of $\theta$, have a discontinuity. Up to a certain critical value of $\theta, V(x ; b)$ is maximized for $b^{*}=0$; at this critical

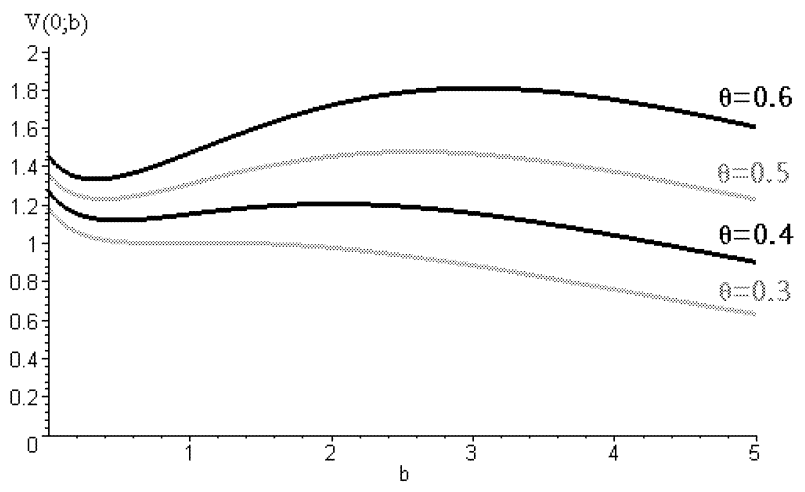

Figure 4: $V(0 ; b)$ for $\theta=0.3,0.4,0.5$ and 0.6 if $p(x)=2\left(\frac{3}{2} e^{-\frac{3 x}{2}}\right)-1\left(3 e^{-3 x}\right), x>0, \alpha=0.1$. 


\begin{tabular}{|c|c|c|c|c|c|c|c|}
\hline$\underline{\theta}$ & .0001 & .001 & .01 & .025 & $\begin{array}{c}\alpha \\
.05\end{array}$ & .10 & .20 \\
\hline .10 & 132.40 & 51.39 & 5.72 & 2.17 & 1.36 & .77 & .24 \\
\hline .20 & 97.62 & 52.14 & 12.10 & 3.79 & 1.85 & 1.02 & .41 \\
\hline .30 & 81.02 & 48.16 & 15.57 & 6.24 & 2.48 & 1.28 & .57 \\
\hline .40 & 71.42 & 44.78 & 17.36 & 8.17 & 3.33 & 1.5 .5 & .71 \\
\hline .50 & 65.19 & 42.20 & 18.29 & 9.55 & 4.33 & 1.83 & .86 \\
\hline .60 & 60.82 & 40.24 & 18.78 & 10.54 & 5.25 & 2.13 & 1.00 \\
\hline .70 & 57.59 & 38.71 & 19.04 & 11.27 & 6.03 & 2.46 & 1.13 \\
\hline .80 & 55.11 & 37.50 & 19.19 & 11.82 & 6.68 & 2.80 & 1.26 \\
\hline .90 & 53.16 & 36.52 & 19.26 & 12.24 & 7.23 & 3.15 & 1.39 \\
\hline 1.00 & 51.58 & 35.71 & 19.30 & 12.58 & 7.69 & 3.51 & 1.52 \\
\hline 1.10 & 50.27 & 35.04 & 19.31 & 12.85 & 8.08 & 3.86 & 1.64 \\
\hline 1.20 & 49.18 & 34.47 & 19.31 & 13.07 & 8.42 & 4.19 & 1.76 \\
\hline 1.30 & 48.26 & 33.99 & 19.31 & 13.26 & 8.71 & 4.50 & 1.89 \\
\hline 1.40 & 47.47 & 33.57 & 19.30 & 13.42 & 8.97 & 4.78 & 2.01 \\
\hline 1.50 & 46.78 & 33.21 & 19.30 & 13.56 & 9.20 & 5.0 .5 & 2.13 \\
\hline 1.60 & 46.18 & 32.89 & 19.29 & 13.68 & 9.40 & 5.29 & 2.25 \\
\hline 1.70 & 45.66 & 32.62 & 19.28 & 13.79 & 9.58 & 5.51 & 2.38 \\
\hline 1.80 & 45.19 & 32.37 & 19.28 & 13.88 & 9.75 & 5.72 & 2.50 \\
\hline 1.90 & 44.78 & 32.16 & 19.28 & 13.97 & 9.90 & 5.90 & 2.62 \\
\hline 2.00 & 44.41 & 31.96 & 19.28 & 14.06 & 10.04 & 6.08 & 2.74 \\
\hline
\end{tabular}

TABLE 3: $b^{*}$ if $p(x)=\frac{1}{3}\left(\frac{1}{2} e^{-\frac{x}{2}}\right)+\frac{2}{3}\left(2 e^{-2 x}\right), x>0$.

\begin{tabular}{rrrrrrrr}
$\underline{\theta}$ & $\mathbf{0 0 0 1}$ & .001 & .01 & .025 & .05 & .10 &. $\mathbf{2 0}$ \\
\hline 10 & 132.43 & 51.78 & 7.15 & 2.19 & .89 & .19 & 0.00 \\
.20 & 97.63 & 52.25 & 12.98 & 4.95 & 2.02 & .81 & .13 \\
.30 & 81.02 & 48.21 & 16.10 & 7.25 & 3.16 & 1.32 & .43 \\
.40 & 71.43 & 44.80 & 17.70 & 8.92 & 4.26 & 1.78 & .68 \\
.50 & 65.19 & 42.22 & 18.52 & 10.12 & 5.22 & 2.22 & .91 \\
.60 & 60.82 & 40.25 & 18.95 & 10.99 & 6.03 & 2.64 & 1.11 \\
.70 & 57.59 & 38.72 & 19.18 & 11.63 & 6.70 & 3.06 & 1.30 \\
.80 & 55.12 & 37.51 & 19.29 & 12.11 & 7.25 & 3.46 & 1.48 \\
.90 & 53.16 & 36.53 & 19.35 & 12.49 & 7.72 & 3.83 & 1.65 \\
1.00 & 51.58 & 35.72 & 19.37 & 12.79 & 8.12 & 4.18 & 1.81 \\
1.10 & 50.27 & 35.04 & 19.38 & 13.03 & 8.46 & 4.49 & 1.96 \\
1.20 & 49.18 & 34.47 & 19.37 & 13.23 & 8.75 & 4.79 & 2.11 \\
1.30 & 48.26 & 33.99 & 19.36 & 13.40 & 9.01 & 5.05 & 2.25 \\
1.40 & 47.47 & 33.57 & 19.35 & 13.55 & 9.24 & 5.30 & 2.40 \\
1.50 & 46.78 & 33.21 & 19.34 & 13.67 & 9.44 & 5.52 & 2.53 \\
1.60 & 46.18 & 32.90 & 19.33 & 13.78 & 9.63 & 5.73 & 2.67 \\
1.70 & 45.66 & 32.62 & 19.32 & 13.88 & 9.79 & 5.92 & 2.80 \\
1.80 & 45.20 & 32.38 & 19.31 & 13.97 & 9.94 & 6.10 & 2.93 \\
1.90 & 44.78 & 32.16 & 19.31 & 14.06 & 10.08 & 6.27 & 3.06 \\
2.00 & 44.41 & 31.97 & 19.30 & 14.13 & 10.21 & 6.42 & 3.18
\end{tabular}

TABLE 4: $b^{o}$ if $p(x)=\frac{1}{3}\left(\frac{1}{2} e^{-\frac{x}{2}}\right)+\frac{2}{3}\left(2 e^{-2 x}\right), x>0$. 


\begin{tabular}{|rrrrrrrrr|}
\hline$\underline{\theta}$ & $\mathbf{0 0 0 1}$ & $\mathbf{. 0 0 1}$ & $\mathbf{. 0 1}$ & $\mathbf{. 0 2 5}$ & $\mathbf{. 0 5}$ & $\mathbf{. 1 0}$ & $\mathbf{. 2 0}$ \\
\hline 10 & 79.20 & 37.69 & 7.24 & 0.00 & 0.00 & 0.00 & 0.00 \\
.20 & 54.91 & 32.88 & 11.21 & 5.16 & 0.00 & 0.00 & 0.00 \\
.30 & 44.10 & 28.52 & 12.40 & 6.77 & 3.53 & 0.00 & 0.00 \\
.40 & 37.95 & 25.54 & 12.59 & 7.63 & 4.43 & 0.00 & 0.00 \\
.50 & 33.96 & 23.42 & 12.45 & 8.07 & 5.05 & 2.58 & 0.00 \\
.60 & 31.15 & 21.85 & 12.21 & 8.29 & 5.47 & 3.03 & 0.00 \\
.70 & 29.07 & 20.65 & 11.96 & 8.39 & 5.76 & 3.39 & 0.00 \\
.80 & 27.45 & 19.70 & 11.72 & 8.43 & 5.97 & 3.67 & 0.00 \\
.90 & 26.17 & 18.92 & 11.50 & 8.43 & 6.11 & 3.90 & 1.96 \\
1.00 & 25.12 & 18.28 & 11.29 & 8.41 & 6.21 & 4.08 & 2.17 \\
1.10 & 24.25 & 17.75 & 11.12 & 8.38 & 6.29 & 4.23 & 2.35 \\
1.20 & 23.51 & 17.29 & 10.95 & 8.35 & 6.34 & 4.36 & 2.51 \\
1.30 & 22.88 & 16.90 & 10.81 & 8.31 & 6.39 & 4.47 & 2.65 \\
1.40 & 22.33 & 16.55 & 10.68 & 8.27 & 6.42 & 4.56 & 2.77 \\
1.50 & 21.86 & 16.25 & 10.57 & 8.24 & 6.44 & 4.63 & 2.88 \\
1.60 & 21.44 & 15.99 & 10.46 & 8.20 & 6.46 & 4.70 & 2.98 \\
1.70 & 21.06 & 15.75 & 10.37 & 8.17 & 6.47 & 4.76 & 3.07 \\
1.80 & 20.73 & 15.54 & 10.28 & 8.14 & 6.48 & 4.81 & 3.15 \\
1.90 & 20.43 & 15.35 & 10.21 & 8.11 & 6.49 & 4.85 & 3.22 \\
2.00 & 20.16 & 15.17 & 10.13 & 8.08 & 6.50 & 4.89 & 3.29 \\
& & & & & & & \\
\hline
\end{tabular}

TABLE $5: b^{*}$ if $p(x)=2\left(\frac{3}{2} e^{-\frac{3 x}{2}}\right)-1\left(3 e^{-3 x}\right), x>0$.

\begin{tabular}{|c|c|c|c|c|c|c|c|}
\hline$\underline{\theta}$ & .0001 & .001 & .01 & .025 & $\begin{array}{c}\alpha \\
.05\end{array}$ & .10 & .20 \\
\hline .10 & 79.21 & 37.76 & 7.68 & 2.97 & 1.14 & 0.00 & 0.00 \\
\hline .20 & 54.91 & 32.90 & 11.41 & 5.54 & 2.75 & 1.04 & 0.00 \\
\hline .30 & 44.11 & 28.53 & 12.50 & 7.00 & 3.90 & 1.82 & 0.00 \\
\hline .40 & 37.95 & 25.54 & 12.65 & 7.78 & 4.70 & 2.43 & 0.00 \\
\hline .50 & 33.96 & 23.42 & 12.49 & 8.17 & 5.25 & 2.92 & 1.26 \\
\hline .60 & 31.15 & 21.86 & 12.24 & 8.37 & 5.63 & 3.30 & 1.57 \\
\hline .70 & 29.07 & 20.65 & 11.98 & 8.45 & 5.88 & 3.61 & 1.83 \\
\hline .80 & 27.45 & 19.70 & 11.73 & 8.48 & 6.06 & 3.86 & 2.06 \\
\hline .90 & 26.17 & 18.92 & 11.51 & 8.47 & 6.19 & 4.06 & 2.26 \\
\hline 1.00 & 25.12 & 18.29 & 11.31 & 8.44 & 6.28 & 4.22 & 2.43 \\
\hline 1.10 & 24.25 & 17.75 & 11.12 & 8.41 & 6.34 & 4.35 & 2.58 \\
\hline 1.20 & 23.51 & 17.29 & 10.96 & 8.37 & 6.39 & 4.46 & 2.71 \\
\hline 1.30 & 22.88 & 16.90 & 10.82 & 8.33 & 6.43 & 4.56 & 2.83 \\
\hline 1.40 & 22.33 & 16.55 & 10.69 & 8.29 & 6.45 & 4.64 & 2.94 \\
\hline 1.50 & 21.86 & 16.25 & 10.57 & 8.25 & 6.47 & 4.70 & 3.03 \\
\hline 1.60 & 21.44 & 15.99 & 10.47 & 8.22 & 6.49 & 4.76 & 3.12 \\
\hline 1.70 & 21.06 & 15.75 & 10.37 & 8.18 & 6.50 & 4.82 & 3.19 \\
\hline 1.80 & 20.73 & 15.54 & 10.29 & 8.15 & 6.51 & 4.86 & 3.26 \\
\hline 1.90 & 20.43 & 15.35 & 10.21 & 8.12 & 6.52 & 4.90 & 3.33 \\
\hline 2.00 & 20.16 & 15.17 & 10.14 & 8.09 & 6.52 & 4.94 & 3.39 \\
\hline
\end{tabular}

TABLE $6: b^{o}$ if $p(x)=2\left(\frac{3}{2} e^{-\frac{3 x}{2}}\right)-1\left(3 e^{-3 x}\right), x>0$. 
value of $\theta, b^{*}$ jumps to a positive value. Figure 4 explains why this happens. For $\alpha=0.1$ the critical value of $\theta$ is 0.4414 . Here $b^{*}$ jumps to the value 2.263. This phenomenon may be due to the shape of $p(x)$.

d) We have seen that $p(x)$ in (8.1) is more dangerous than $p(x)$ in (8.2), and that $p(x)=e^{-x}$ is somewhere in between. One might think that for a fixed $\alpha$ and $\theta$, the three values of $b^{*}$ (or $b^{o}$ respectively) would always be in the same order. But this is not the case.

\section{ACKNOWLEDGMENT}

Hans U. Gerber and Elias Shiu acknowledge with thanks the support from the Patrick Poon Lecture Series Programme in Actuarial Science and the Principal Financial Group Foundation. The authors are grateful to the referees for their comments.

\section{REFERENCES}

Azcue, P. and Muler, N. (2005) Optimal reinsurance and dividend distribution policies in the Cramér-Lundberg model. Mathematical Finance, 15, 261-308.

Borch, K. (1974) The Mathematical Theory of Insurance. Lexington Books, Lexington, MA. BorCH, K. (1990) Economics of Insurance. North Holland, Amsterdam.

BüHlmann, H. (1970) Mathematical Methods in Risk Theory. Springer-Verlag, Berlin Heidelberg New York.

Burden, R.L., Douglas Faires, J. and Reynolds, A.C. (1981) Numerical Analysis, Second Edition. Prindle, Weber and Schmidt, Boston, MA.

De FinetTi, B. (1957) Su un'impostazione alternativa della teoria collettiva del rischio. Transactions of the XVth International Congress of Actuaries, 2, 433-443.

Dickson, D.C.M. (2005) Insurance Risk and Ruin. Cambridge University Press.

Dickson, D.C.M. and WATERs, H.R. (2004) Some optimal dividends problems. ASTIN Bulletin, 34, $49-74$.

GERBER, H.U. (1979) An Introduction to Mathematical Risk Theory. Huebner Foundation, Philadelphia.

Gerber, H.U. and Shiu, E.S.W. (2006) On optimal dividend strategies in the compound Poisson model. Forthcoming in North American Actuarial Journal 10(2).

Lin, X.S., Willmot, G.E. and Drekic, S. (2003) The classical risk model with a constant dividend barrier: analysis of the Gerber-Shiu discounted penalty function. Insurance: Mathematics and Economics, 33, 551-566.

Paulsen, J. and GJessing, H.K. (1997) Optimal choice of dividend barriers for a risk process with stochastic return on investments. Insurance: Mathematics and Economics, 20, 215-223.

Schmidui, H. (2007) Stochastic Control in Insurance. Springer-Verlag, London, forthcoming. 
HANS U. GeRber

Distinguished Visiting Professor at The University of Hong Kong

Ecole des hautes études commerciales

Université de Lausanne

CH-1015 Lausanne, Switzerland

E-mail: hgerber@unil.ch

ELIAS S.W. SHIU

Visiting Professor at The University of Hong Kong

Department of Statistics and Actuarial Science

The University of Iowa

Iowa City, Iowa 52242-1409, USA

E-mail: eshiu@stat.uiowa.edu

NATHANIEl SMith

Ecole des hautes études commerciales

Université de Lausanne

CH-1015 Lausanne, Switzerland

E-mail:nsmith@unil.ch 